\title{
Catecholamine Toxicity After Craniotomy and Evacuation of the Abscess
}

\author{
Wulan Fadinie*, Ongta Gibson Sirait \\ Department of Anesthesiology and Intensive Care, Adam Malik Central General Hospital, Medan, North Sumatera, Indonesia
}

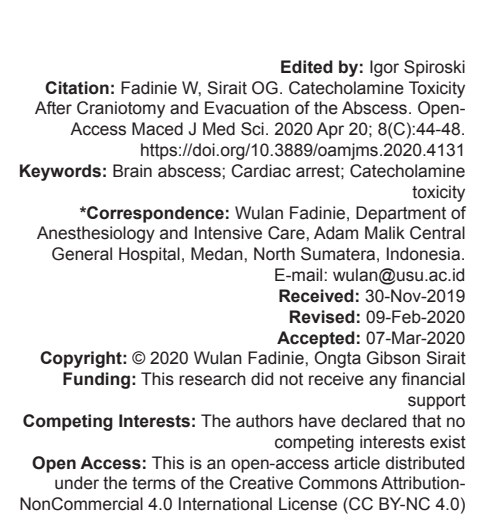

\begin{abstract}
BACKGROUND: Brain abscess is a focal infection in the brain parenchyma, may be through direct extension or hematogenous. Brain abscess is a rare complication of neonatal meningitis that occurs in $1-4 \%$ of all cases. In infants and toddlers, bacterial meningitis or bacteremia is the major cause.

CASE REPORT: In this case, the patient is diagnosed with a brain abscess since birth. According of the history, physical examination, and investigations, it was concluded the diagnosis of epidural abscess with abscess evacuation craniotomy and PS ASA 2 (leukocytosis) with GA-ETT anesthesia. The operation is carried out with a duration of $4 \mathrm{~h}$. Vital sign monitoring obtained blood pressure 108-125/62-90 mmHg, heart frequency 90-120 times/min, and $99 \%$ oxygen saturation. When in the recovery room, the patient experiences cardiac arrest. Sudden cardiac arrest in children is a rare event. This is thought to result from catecholamine toxicity. Patient is treated as resuscitation in accordance with the algorithm of cardiac arrest in children. Patient experienced a response of spontaneous circulation and performed vital sign monitoring.
\end{abstract}

CONCLUSION: Increasing levels of endogenous catecholamines occur acutely to provide short-time adaptation to stressful conditions. Catecholamine toxicity requires multidisciplinary management.

\section{Introduction}

Brain abscess is a focal infection of the brain which is a rare complication of neonatal meningitis, can diagnose using radiological examinations such as ultrasonography (USG), computed tomography (CT), magnetic resonance imaging (MRI), as well as microbiological techniques along with more effective antibiotics have improved treatment. In infants and toddlers, the main cause of meningitis is bacterial so that fast and long-term antimicrobial therapy is the main treatment; this antimicrobial administration is often associated with surgical drainage. This change in management has significantly improved the prognosis of patient with brain abscesses over the past 50 years. The management of brain abscess therapy requires multidisciplinary management.

While in the post-operative room, the patient experiences cardiac arrest, which is suspected to be due to catecholamine toxicity after evacuation abscess of brain. Catecholamines, released from the central nervous system and sympathetic, function as hormones and neurotransmitters play an important role in the cardiovascular system.

Sudden cardiac arrest that occurs in children is rare. Doctors must understand the conditions that can cause cardiac arrest. This article reviews the most common causes of sudden cardiac arrest in a pediatric population. We discuss the latest management recommendations for dealing with various causes of sudden cardiac arrest in the pediatric population.

Increasedlevelsofendogenouscatecholamines occur under stressful conditions, known as fight-or-fly. Likewise, administration of exogenous catecholamines is a savior in clinical situations related to decrease cardiac output and/or hypotension. In contrast to these short-term goals, a continued increase in catecholamines can be dangerous to the cardiovascular system characterized by significant cardiotoxicity, such as in chronic heart failure, pheochromocytoma, stressstimulated cardiomyopathy ("takotsubo"), and patient receiving prolonged therapy with high doses. In the latter condition, the administration of isoproterenol b-adrenoceptors to laboratory animals has been used for decades as a model for assessing heart injury [1].

$$
\text { Catecholamine poisoning requires }
$$
interdisciplinary discipline, especially in the management of complex catecholamines and the selection of appropriate resuscitation. A recent literature review conducted by Whitelaw et al. examined that mortality was significantly higher in those who did not receive alpha-blockade for the treatment of the catecholamine crisis compared with those who received treatment 
with alpha-blockade (99\% vs. $40 \%$ ). Of course, the use of alpha-blockade may have been contraindicated in some of the cases reviewed because of hypotension accompanied by cardiogenic shock and/or thinning of the intravascular wall. As explained in our case, monitoring proper fluid status can guide proper fluid resuscitation. The researchers recommend that patient should be monitored in the intensive unit accompanied by nurse expertise and access to mechanical circulation if needed. The same researchers have also shown that initial test doses of short-acting alpha-blockers, such as phentolamine, provide information about cardiovascular reserves in these patient and appropriate fluid resuscitation allows optimal alpha-blockade [2].

\section{Case Report}

A 9-month-old male patient with a body weight of $6.1 \mathrm{~kg}$ entered the emergency room with a reduction in awareness by diagnosing brain abscesses. Patient is diagnosed with a patient's brain abscess from birth. A history of fainting was denied, a history of seizures was denied. A history of fever is denied. History of trauma is denied. Patient experiences difficulties during labor.

From the physical examination in the room on July 25, 2018, airway clear was found, no snoring, gurgling, and crowing were found, with a frequency of breathing 52 times/min, vesicular breath sounds, no additional breath sounds and 99\% oxygen saturation. Circulation is found akral warm, red, dry, with heart frequencies 142 times/min, regular, with strong pulse pressure, and sufficient volume. The patient's consciousness was compos mentis, GCS 15, with isochoric pupils $\varnothing 3 \mathrm{~mm}$ right and left and positive light reflexes. Positive urine output, abdomen soepel, and peristaltic are positive normal. No edema and fractures of the extremities were found.

Laboratory tests on July 24, 2018 obtained hemoglobin $(13.4 \mathrm{gr} / \mathrm{dl})$, hematocrit $(42 \%)$, leukocytes $(13,910 \mathrm{gr} / \mathrm{dl})$, platelets $(393,000 / \mathrm{ul})$, sodium (137 $\mathrm{mmol} / \mathrm{L})$, potassium $(4.3 \mathrm{mmol} / \mathrm{L})$, chloride (106 mmol/L), blood glucose level (114 mg/dl), urea (40 mg/dl), and creatinine $(0.27 \mathrm{mg} / \mathrm{dl})$.

Radiological examination revealed chest radiographs within normal limits (Figure 1).

Radiological examination obtained a CT scan of the head with a brain abscess in the right hemisphere (Figure 2).

Physical examination and investigations, the patient was diagnosed with an epidural abscess that requires craniotomy treatment for the evacuation of the abscess and the patient was classified as PS ASA 2 (leukocytosis) by choosing GA ETT anesthesia techniques (Figure 3).

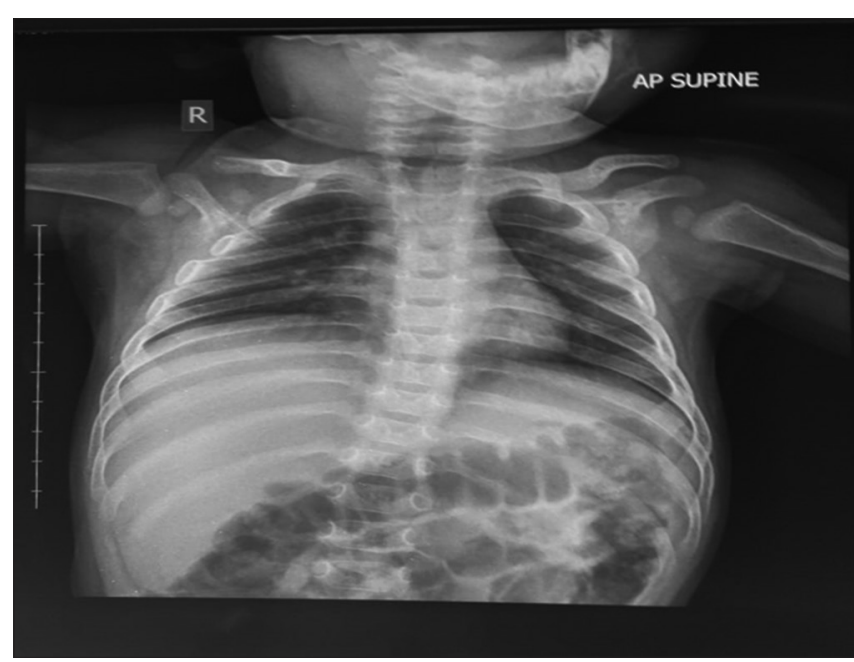

Figure 1: Chest $X$-ray

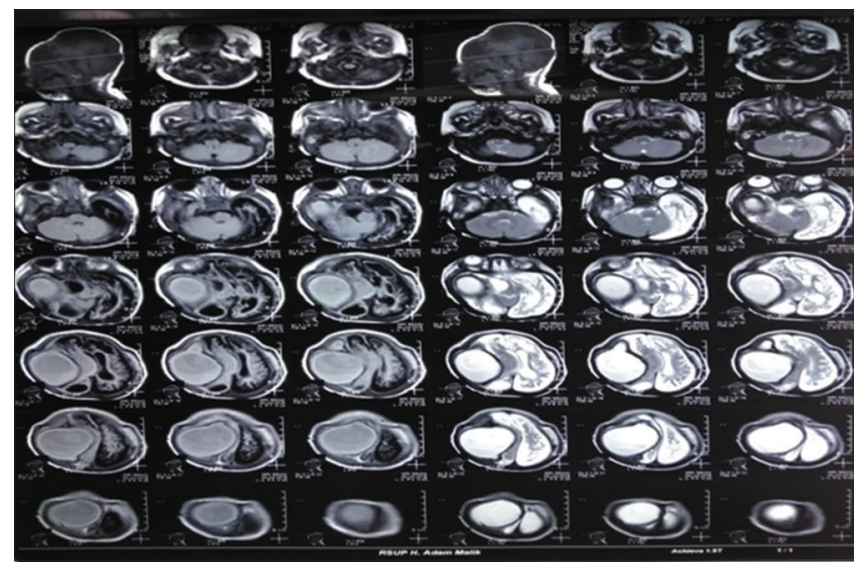

Figure 2: X-ray head CT scan

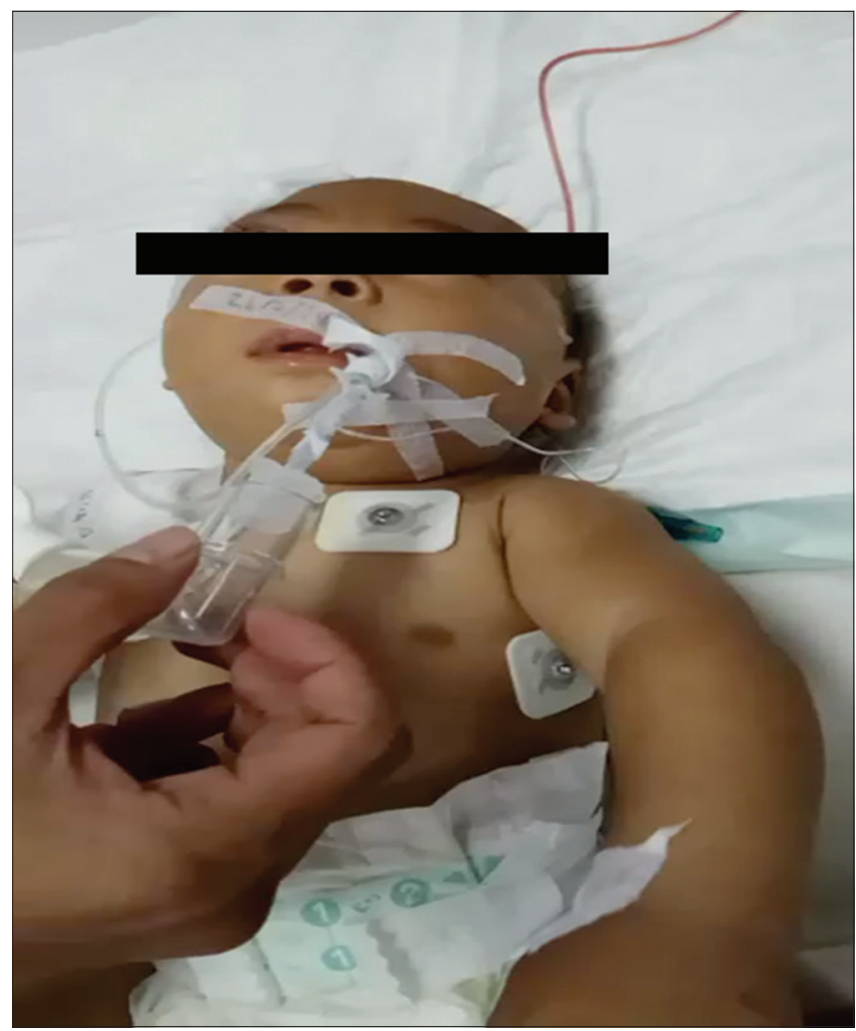

Figure 3: Clinical appearance of the patient 
Providing education to the patient's family $24 \mathrm{~h}$ before surgery, authorizing anesthetic action and completing approval for surgery, fasting patient $4 \mathrm{~h}$ before surgery, IV Line $24 \mathrm{G}$ installation and ensuring smoothness, Cefazolin injection $200 \mathrm{mg} 1 \mathrm{~h}$ before surgery, preparation 1 bag of blood, shaved head hair, oral, and personal hygiene. Anesthesia technique: active suction, Pre-oxygenated $\mathrm{O}_{2} 6 \mathrm{~L} / \mathrm{min}$ in $3-5 \mathrm{~min}$. Midazolam injection $1 \mathrm{mg}$ (intravenously), Fentanyl injection $12 \mathrm{mcg}$ (intravenously), Sellick maneuver, and Propofol Induction $15 \mathrm{mg}$ (intravenously) with negative eyelid evaluation, Rocuronium injection $8 \mathrm{mg} /$ (intravenously) with evaluation until positive sleep apnea. Maintenance given water: $\mathrm{O}_{2}=2 \mathrm{~L}: 2 \mathrm{~L}$, Isoflurane $0.8-1 \%$.

The duration of the operation takes $4 \mathrm{~h}$. Monitoring vital signs obtained blood pressure 108$125 / 62-90 \mathrm{mmHg}$, heart frequency 128-146 times/min, and oxygen saturation of $99 \%$.

Fluid balance during pre-operative with lactated ringer fluid $(100 \mathrm{ml})$, durante operation with lactated ringer $(540 \mathrm{ml})$, bleeding $+100 \mathrm{ml}$, evaporation plus maintenance: $(2+8) \times 6.1 \mathrm{~kg}=61 \mathrm{ml} / \mathrm{h}$. Urine output is $10 \mathrm{ml} / \mathrm{h}$.

While in the post-operative room, the patient experiences cardiac arrest, which is suspected to be due to catecholamine toxicity after evacuation abscess of brain. Patient is given a pediatric cardiac arrest algorithm, then responds of spontaneous circulation and replaces vital signs.

Therapy after surgery bed rest, head $30^{\circ}$, given $\mathrm{O}_{2} 3 \mathrm{~L} / \mathrm{min}$ through nasal cannula, TPN diet, IVFD RL 30 drops micro/min, ceftriaxone injection $200 \mathrm{mg} / 12 \mathrm{~h}$ (intravenously), $100 \mathrm{mcg}$ injection of fentanyl dissolved in $50 \mathrm{ml}, 0,9 \% \mathrm{NaCl}$ was given $1 \mathrm{ml} / \mathrm{h}$, ranitidine injection $10 \mathrm{mg} / 12 \mathrm{~h}$ (intravenously).

\section{Discussion}

Brain abscesses begin with lesions consistent with cerebritis. This can last for several weeks followed by edema which can create a mass effect. The tissue then becomes necrotic and is surrounded by granulation tissue.

Brain abscessescan be polymicrobials involving aerobic and anaerobic organisms. Common organisms: Most are anaerobic, streptococcal, staphylococcal, and Bacteroides fragilis. Proteus is common in neonates. Predisposing factors: Brain abscess tends to occur in children with cyanotic congenital heart disease, sepsis, endocarditis, after neurosurgical procedures, lung infections, paranasal sinus infections or trauma or otomastoid areas. Children who are immune deficient/ immunosuppressed are also at risk.

\section{Clinical characteristics}

The emergence of headaches and fever.

Focal neurological signs may be present. Vomiting, confusion, nuchal stiffness, and seizures may also be seen. Diagnostic tests: Blood culture can play a role in isolating organisms. Neuroimaging is the key and contrast is needed for a definitive diagnosis.

\section{Imaging characteristics}

(1) CT: Low attenuation and thin walls with a slight increase in attenuation, and increased wall contrast. (2) MRI: Abscess wall is isointense at T1 and hypointense at T2. The center is isointense to hypointense on T1 and hyperintense on T2. Enhanced ringing comes with contrast. Diffusion studies are the key to deciphering abscesses from cystic or necrotic tumors. Abscesses will have limited diffusion (hyperintense on DWI, and hypointense on ADC). Diffusion abnormalities will become normal 1 to 2 weeks after treatment. Treatment: Don't delay antibiotics. The first control increases ICP. Drainage and surgical incisions can play the role followed by antibiotics depending on the stage of the abscess. In the early stages or if there are multiple abscesses, medical therapy is usually the first choice. Serial imaging studies will help measure responses to medical therapy. Neurological sequelae: Chronic seizures/epilepsy.

Catecholamines released from the adrenal medulla and from the central and sympathetic nervous system, function as hormones and neurotransmitters play an important role in the cardiovascular system. A very high increase in catecholamines causes toxicity that will result in cardiac arrest. Structurally, catecholamines include the dihydroxybenzene ring (catechol) and the nitrogen group (amine). They are produced from the amino acid L-tyrosine through hydroxylation, decarboxylation, and successive methylation steps which result in sequential formation of dihydroxyphenylalanine (L-Dopa), dopamine, norepinephrine (NE, noradrenaline), and epinephrine (Epi, adrenaline) [1].

Catecholamines in high concentrations produce myocardial damage in some mammalian species. Histological changes are similar to those found in patient given large amounts of pressor agents and in those who develop pheochromocytomas. They include myofiber necrosis, myofibrillar degeneration, and mononuclear leukocyte infiltration. Heart function is significantly impaired. Endogenous release of catecholamine can also cause myocardial injury in rabbit patient infused with tyramine. Anatomical and functional abnormalities described in various models of catecholamine cardiomyopathy are summarized. Some of the main theories about pathogenesis are reviewed. The latest data show that the generation of free radicals originating from two is involved discussed [1]. 
The pathophysiological events triggered by an increase in $[\mathrm{Ca2+} \mathrm{M}$ are significantly strengthened by oxidative stress which develops on sustained exposure to high levels of catecholamines, due to the mechanism identified below. First, MAO-dependent oxidative deformation of catecholamines forms hydrogen peroxide $\left(\mathrm{H}_{2} \mathrm{O}_{2}\right)$, which can be converted into highly reactive hydroxyl radicals $\left(\mathrm{OH}^{-}\right)$through metal catalysis (Fenton chemistry). Second, activation of A1-adrenoceptors by catecholamine induces activation of NADPH oxidase, with the next generation of superoxide $\left(\mathrm{O}_{2}-\right)$ anion radicals in cardiac myocytes. Third, and most importantly, catecholamines are easily oxidized to toxic compounds called "aminochromes" (because of their colored appearance in solution). This process occurs spontaneously at a low level (autoxidation), but this is markedly accelerated in the presence of oxidants and free radicals such as $\mathrm{O}_{2}-$, redox metals (especially iron and copper) and by enzymatic catalysis (mainly by xanthine oxidase, myeloperoxidase, and cytochrome oxidase) [2].

In short, sustained high level catecholamines can cause major toxic effects on the myocardium, which cause morphological changes similar to those produced by myocardial infarction, including cell death especially cardiomyocytes and progressive myocardial focal fibrosis. The toxicity comes from a variety of catecholamine actions in the heart, the most significant being excess calcium, oxidative stress, and mitochondrial dysfunction [1], [4].

Clinical manifestations caused in the form of paroxysmal hypertension. For example, in patient with pheochromocytoma, episodes of hypertension are usually sudden onset and often accompanied by physical symptoms, such as headache, dizziness, nausea, diaphoresis, chest pain, and palpitations. The frequency of these episodes ranges from every day to less than once per month, and the duration of each episode can range from minutes to days. This disorder is more common in women. A potential explanation for this can be increased activation of specific cardiac sympathetic nervous systems in women compared to men [5].

There is no specific therapeutic approach for catecholamine-induced cardiomyopathy until now. The management of catecholamine-induced cardiomyopathy should include a reduction in sympathetic activation using $\alpha$-and $\beta$-adrenergic receptor inhibitors and administration of diuretics in case of excessive volume. Emergency management of arrhythmias, pulmonary edema, and cardiogenic shock based on conventional symptomatic treatment, such as antiarrhythmics and inotropics, vasoactive electric shock, and/or mechanical circulation support [1].

\section{Conclusion}

Brain abscess is a focal infection in the brain parenchyma, may be through direct extension or hematogenous. Brain abscess is a rare complication of neonatal meningitis that occurs in $1-4 \%$ of all cases. Early diagnosis using radiological modalities such as USG, CT, MRI, and microbiological techniques together with more effective antibiotics has improved treatment. In infants and toddlers, bacterial meningitis, or bacteremia is the major cause, and in older children, immunosuppression, and cyanotic congenital heart diseases are common predisposing factors. The therapeutic management of brain abscesses involves a multidisciplinary team. A prompt and long-term antimicrobial therapy is the mainstay of treatment, often associated with surgical drainage. These changes in the management have significantly improved the prognosis of patient with brain abscesses over the past 50 years.

Sudden cardiac arrest in children is a rare event. However, sudden cardiac arrest of a child is a devastating event. Clinicians should be aware of conditions associated with sudden cardiac arrest in the pediatric population. This article reviews the most common causes of sudden cardiac arrest in the pediatric population. We review recommendations for screening and diagnosis. We also briefly discuss current management recommendations for the various causes of sudden cardiac arrest in the pediatric population.

Catecholamine toxicity can cause cardiac arrest. Increased levels of endogenous catecholamines occur acutely to provide short-term adaptation to stressful conditions, known as the fight-or-fly. There is no specific therapeutic approach for catecholamineinduced cardiomyopathy until now. The management of catecholamine-induced cardiomyopathy should include a reduction in sympathetic activation using $\alpha$-and $\beta$-adrenergic receptor inhibitors and administration of diuretics in case of excessive volume. Emergency management of arrhythmias, pulmonary edema, and cardiogenic shock are based on conventional symptomatic treatment, such as antiarrhythmic and inotropics, vasoactive electric shock and/or mechanical circulation support.

Catecholamines and their oxidation products cause a direct toxic effect on the myocardium. Catecholamines also exert a receptor-mediated effect on the myocardium. Catecholamine-mediated myocardial stunning has been implicated in the pathogenesis of stress-induced cardiomyopathy. In this case, massive abscess evacuation in patient can cause catecholamine toxicity. 


\section{References}

1. Liaudet L, Calderari B, Pacher P. Pathophysiological mechanisms of catecholamine and cocaine-mediated cardiotoxicity. Heart Fail Rev. 2014;19(6):815-24. https://doi. org/10.1007/s10741-014-9418-y

PMid:24398587

2. Casey RT, Challis BG, Pitfield D, Mahroof RM, Jamieson N, Bhagra CJ, et al. Management of an acute catecholamineinduced cardiomyopathy and circulatory collapse: A multidisciplinary approach. Endocrinol Diabetes Metab Case Rep. 2017;1(1):1-6. https://doi.org/10.1530/edm-17-0122 PMid:29147570
3. Garcha AS, Cohen DL. Catecholamine excess: Pseudopheochromocytoma and beyond. Adv Chronic Kidney Dis. 2015;22(3):218-23. https://doi.org/10.1053/j.ackd.2014.11.002 PMid:25908471

4. Jia X, Guo X, Zheng Q. Perioperative management of paraganglioma and catecholamine-induced cardiomyopathy in child-a case report and review of the literature. BMC Anesthesiol. 2017;17(1):142. https://doi.org/10.1186/s12871-017-0433-0 PMid:29041921

5. Jiang JP, Downing SE. Catecholamine cardiomyopathy: Review and analysis of pathogenetic mechanisms. Yale $\mathrm{J}$ Biol Med. 1990;63(6):581-91.

6. Kathrine BS, Jurriaan MP, Patricia LM, Zelime EM. Handbook of Pediatric Neurology. Boston: Wolters Kluwer; 2014. p. 515. 